TAO, Vol. 15, No. 4, 589-607, November 2004

\title{
Improvement of GPS/MET Ionospheric Profiling and Validation Using the Chung-Li Ionosonde Measurements and the IRI model
}

\author{
Lung-Chih Tsai ${ }^{1,2, *}$, and Wei-Hsiung Tsai ${ }^{2}$
}

(Manuscript received 28 November 2003, in final form 26 July 2004)

\begin{abstract}
The retrieval processes of ionospheric profiling from radio occultation observations usually assume spherical symmetry of electron density distribution at the locality of occultation and use the Abel integral transform to invert the measured total electron content (TEC) values. In this paper, we have considered the effect of large-scale horizontal gradient and/or inhomogeneous electron density and developed a compensation procedure for measured TEC values through several close-up occultation observations. The compensated TEC values are then used to yield electron densities through the Abel inversion. In order to assess the accuracy of GPS/MET ionospheric electron density retrievals, coincidences of the Chung-Li digisonde data with observed GPS/MET occultations have been examined. From 232 matches during the GPS/MET mission, the rms $f_{0} F_{2}$ differences between the digisonde measurements and the GPS/MET retrievals are improved from $1.58 \mathrm{MHz}$ to $1.41 \mathrm{MHz}$. Further validation of the improved retrieval processes has also been investigated by means of over thirty-eight thousand simulated occultation observations within a reference GPS/MET experiment using the International Reference Ionosphere (IRI) 2001 model. The results show successful improvement of overestimates (underestimates) on low (high) retrieved $f_{0} F_{2}$.
\end{abstract}

(Key words: Radio occultation, GPS/MET, Ionospheric profiling, Abel inversion, Total electron content (TEC))

\footnotetext{
${ }^{1}$ Center for Space and Remote Sensing Research, National Central University, Chung-Li, Taiwan, $\mathrm{ROC}$

2 Institute of Space Science, National Central University, Chung-Li, Taiwan, ROC

* Corresponding author address: Prof. Lung-Chih Tsai, Center for Space and Remote Sensing Research, National Central University, Chung-Li, Taiwan, ROC; E-mail: Ictsai@csrsr.ncu.edu.tw
} 


\section{INTRODUCTION}

The radio occultation observation technique has been successfully used to remote sense the atmosphere and other properties of planets since the 1960s (Kilore et al. 1965; Fjeldbo and Eshleman 1969). In 1993 the University Corporation for Atmospheric Research (UCAR) organized a proof-of-concept experiment on a LEO satellite (the Microlab-1 satellite) at a nearly circular orbit of $\sim 735 \mathrm{~km}$ altitude and a $\sim 70^{\circ}$ inclination angle to receive GPS signals and to demonstrate active limb sounding of the Earth's atmosphere and ionosphere by the radio occultation technique. The main objectives of this GPS-LEO occultation mission were numerical weather prediction and long-term monitoring of the Earth's climate and thus it was termed the GPS/Meteorology (GPS/MET) program (Zou et al. 1995; Ware et al. 1996; Kursinski et al. 1997; Rocken et al. 1997). GPS/MET has also been able to sound the ionosphere from the LEO satellite orbit altitude to $\sim 60 \mathrm{~km}$ below the $\mathrm{D}$ and $\mathrm{E}$ regions and provide profiles of ionospheric electron density from measurements of ray-path bending angle or total electron content (TEC). (Hajj and Romans 1998; Hocke and Igarashi 2002a). A GPS-LEO occultation geometry is depicted as Fig. 1. When a GPS satellite sets or rises behind the Earth's ionosphere as seen by a LEO satellite, the GPS radio signals are received by a receiver on the LEO satellite. Each occultation therefore consists of a set of limb-viewing links with tangent points ranging from the LEO satellite orbit altitude to the surface of the Earth. These retrieval processes generally assume spherical symmetry of electron density (or refractivity) at the locality of occultation and use the Abel integral transform to invert the bending angle or TEC data to be one-dimensional results as a function of tangent point altitude. It is obvious that this assumption of spherical symmetry in the ionosphere (and the atmosphere) is almost never true. Sokolovskii (1990) firstly proposed a method of meteorological parameter inversion which takes into account the horizontal heterogeneity of the atmosphere. There are several works which examine and estimate the accuracy of retrieved GPS/MET electron density profiles obtained with the Abel inversion by comparing them with vertical ionosonde data (Hajj and Romans 1998; Schreiner et al. 1999; Tsai et al. 2001). All authors obtained fractional rms differences from $20 \%$ to $40 \%$ in $N_{m} F_{2}$ implying differences from $10 \%$ to $20 \%$ in $f_{0} F_{2}$ and they believed the dominant error in the Abel inversion is due to the locally spherical symmetry assumption imposed on the ionosphere.

Instead of one-dimensional ionospheric profiling with two-dimensional (or even threedimensional) reconstruction in occultation data analysis, several techniques such as tomography (Rius et al. 1997, 1998; Hajj et al. 2000; Tsai et al. 2002) and ionospheric data assimilation (Hajj et al. 2000; Dymond and Thomas 2001) have been implemented for the GPS/MET experiment. Hocke and Igarashi (2002b) also proposed a 2-D recovery method, which can compute the electron density field within a meridian plane at all latitudes and from the top layer of the LEO satellite altitude to the base of the ionosphere. However, because there is insufficient occultation data, two-dimensional reconstruction can only depict large-scale horizontal electron density variations and observe large-scale ionospheric features with rough accuracy and resolution. Meanwhile, with the lack of ionospheric observational data those techniques usually summarize a sufficient amount of occultation data from several (NOT only one) LEO satellite revolutions of the Earth and thus trade off temporal and/or spatial resolutions, 


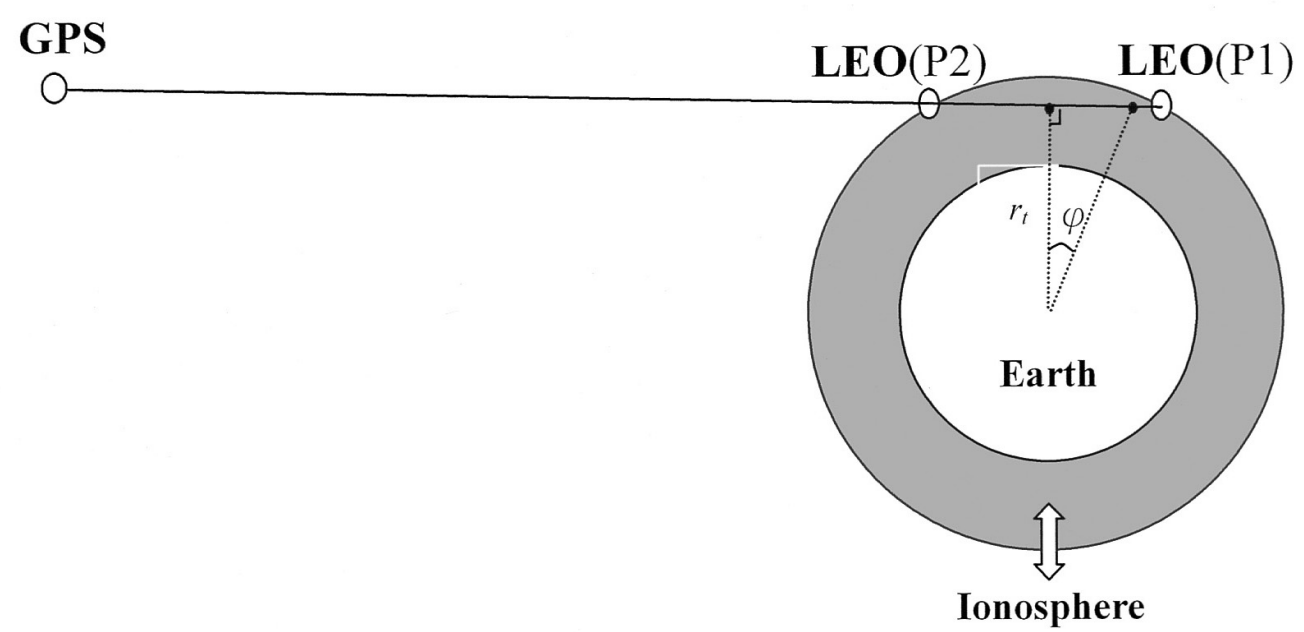

Fig. 1. Illustration of the geometry of the GPS-LEO occultation problem for ionosphere observations.

especially vertical resolution. For the practical Abel inversion technique, the theoretical spatial resolutions are mainly dominated by diffraction effects to the Fresnel zones (Kursinski et al. 1997) and have a very high vertical resolution of close to zero to $2 \mathrm{~km}$ but a horizontal resolution of $200-500 \mathrm{~km}$ depending on the impact distance of radio occultation observations.

With respect to one-dimensional ionospheric profiling (Straus 1999; Hajj et al. 2000) a constrained-gradient Abel inversion method has been presented, which uses earlier simulated horizontal gradients from other ancillary data and constrains the practical Abel inversion considering the horizontal asymmetry effect on electron density retrieval. Three different types of ancillary data (in-situ plasma density, nadir-viewing extreme ultraviolet airglow, and vertical TEC maps derived from ground-based GPS receivers) have been simulated using the RIBG ionospheric model (Reilly 1993) and provide "known" horizontal gradients. The simulation results represented significant improvement using any of the three gradient correction techniques. However, when realistic errors of the ancillary data are considered, the obtained improvement is not as great as previously estimated (Schreiner et al. 1999; Hajj et al. 2000). In the following we also propose to regularize an improvement of the Abel inversion method for ionospheric profiling. The inverse problem is solved by using the Abel inversion based on path TEC values obtained within occultation observations in two different ways: (1) as in the earlier works (Schreiner et al. 1999; Tsai et al. 2000), calculated by the calibrated path TECs between the occulting LEO satellite positions and the corresponding auxiliary positions with the same impact distances, and (2) compensating the previously calibrated TECs with the line integral of the electron density differences between the previous Abel inverted electron densities of the targeted occultation observation and the interpolated electron densities from nearby ionospheric profiles retrieved from close-up occultations. In section 2, we overview iono- 
spheric electron density profiling from GPS radio occultation observations through the Abel inversion. In section 3 , an improved Abel inversion scheme is presented and discussed. The retrieved $f_{0} F_{2}$ results before and after improvement have been examined and evaluated by an ionospheric sounder (the Chung-Li digisonde at $24.6^{\circ} \mathrm{N}, 121.0^{\circ} \mathrm{E}$ ) in section 4 . In section 5, further validation of the improved retrieval processes has also been investigated by means of over thirty-eight thousand simulated occultation observations within the reference GPS/MET experiment through the IRI 2001 model. We draw our conclusions in section 6.

\section{IONOSPHERIC PROFILING USING THE ABEL INVERSION TECHNIQUE}

In this paper, electron density profiles have been derived by the Abel inversion through the computation of TEC under the assumption of straight-line propagation to approach a near real-time process. The electron densities can also be computed by precise refractivity values inverted by the same Abel inversion through measured bending angles, which can be calculated from the observed excess Doppler frequency shifts using the Bouguer's rule (i.e., Snell's law with an assumption of spherical symmetry) and optical geometry properties (Hajj and Romans 1998; Schreiner et al. 1999). The GPS/MET TEC values or the corresponding ionospheric delay can be derived from signal phase measurements, which can approach 0.01 TEC units when Anti-Spoofing (AS) is off or with enhanced codeless GPS receivers. Formally phase measurements in units of distance (or excess phase) can be modeled as:

$$
S_{i}=-\frac{c}{f_{i}} \Phi_{i}=m_{i} \lambda_{i}+\rho+\delta_{i}+40.3 \frac{T E C_{i}}{f_{i}^{2}}+C^{T}+C^{R}+\varepsilon_{i}
$$

where $i=1$ or 2 for $\mathrm{L} 1$ or L2 frequencies $\left(f_{1}=1575.42 \mathrm{MHz}\right.$ and $\left.f_{2}=1227.60 \mathrm{MHz}\right)$, respectively; $\Phi$ is the recorded phase in cycles; $c$ is light speed in free space; $m$ is an integer which is constant over a connected arc; $\lambda$ is the operating wavelength; $\rho$ is the true range between the targeted GPS and LEO satellites; $\delta$ is the extra delay due to the lower neutral atmosphere but is zero here for ionospheric observations; TEC is the total electron content between GPS and LEO; $C^{T}$ and $C^{R}$ are transmitter and receiver clock errors, respectively, and $\varepsilon$ is phase measurement noise. We note that, at GPS frequencies, bending in the ionosphere is small enough so that ray separation from straight-line propagation for observations in LEO is only about a few kilometers or less, which is much smaller than typical vertical scales of the electron density in the ionosphere. Even during the daytime and near solar maximum, the absolute magnitude of the bending angle does not exceed $0.03^{\circ}$ for both of L1 and L2 GPS frequencies (Hajj and Romans 1998; Schreiner et al. 1999) in the F-region. Therefore, because bending angles at GPS frequencies are negligible for monitoring the F2 layer, then we can assuming straight-line propagation is valid and will allow the computation of TEC that is the same whether calculated from either $S_{1}, S_{2}$, or from $S_{1}-S_{2}$ as given as the following: 


$$
T E C=\int n_{e} d l=-\frac{f^{2}}{40.3} \int(n-1) d l=-\frac{f_{1}^{2} S_{1}}{40.3}=-\frac{f_{2}^{2} S_{2}}{40.3}=\frac{\left(S_{1}-S_{2}\right) f_{1}^{2} f_{2}^{2}}{40.3\left(f_{1}^{2}-f_{2}^{2}\right)},
$$

where TEC is in el. $\mathrm{m}^{-2}, n_{e}(n)$ is ionospheric electron density (refractive index), and $S_{1}$ and $S_{2}$ are in meters. In this study the limb-viewing TEC values are derived by dual frequency excess phases; the excess phases were obtained from $0.1 \mathrm{~Hz}$ GPS carrier phase change data but interpolated to 300 values from top to bottom of occultation using a cubic spline fit. We further assume that the ionosphere distribution above the LEO orbit altitude is static and there is local spherical symmetry over the duration of an occultation and can thus determine the "calibrated" path TEC between an occulting LEO position and its calibration position (having the same LEO altitude but at the other side of occultation along a "straight" GPS-LEO link) to be the limb-viewing TEC difference obtained from the original occulting LEO position and another auxiliary LEO position also (at the other side of occultation also and having the same impact distance), illustrated as P1 and P2 in Fig. 1. We note that, when the LEO orbit and GPS-to-LEO occultation links are coplanar, the corresponding calibration position and auxiliary position could coincide. Under the assumption of straight-line propagation, the calibrated TEC (TEC') at a tangent point radial distance $r_{t}$ is related to electron density through the following integral:

$$
T E C^{\prime}\left(r_{t}\right)=T E C(P 1)-T E C(P 2) \cong 2 \int_{r_{t}}^{r_{L E O}} \frac{r n_{e}(r)}{\sqrt{r^{2}-r_{t}^{2}}} d r
$$

The integral equation can also be inverted by using an Abel integral transform given by Tricomi (1985). Applying the Abel inversion, as is done through bending angles with an assumption of locally spherical symmetry, the electron density can then be given by the following integral equation:

$$
n_{e}\left(r_{t}\right)=-\frac{1}{\pi} \int_{r_{t}}^{r_{L E O}} \frac{d T E C^{\prime}(r) / d r}{\sqrt{r^{2}-r_{t}^{2}}} d r
$$

We note that the derived electron density precision is limited by the accuracy with which the GPS signal phases can be measured. However, in practice, inhomogeneous electron density in the horizontal direction for a given occultation is believed to be the main source of error in the Abel inversion. Such errors are increased when the radio baseline length is increased or limb sounding is propagated through the lower ionospheric layers, e.g., the D- and E-layers, and even the low and middle atmosphere. Meanwhile, the ellipsoidal shape of the Earth also produces small departures from spherical symmetry in the refractive index or electron density field used in the Abel inversion. Other errors are caused by the assumption of straight-line propagation, which however can be ignored from LEO satellite altitudes through the bottom of the F layer. 


\section{THE IMPROVED ABEL INVERSION PROCESS THROUGH "COMPENSATED" TEC VALUES}

The Microlab-1 spacecraft was equipped with a single antenna that tracked the GPS satellites in view of setting (or rising for a few percentages) behind the Earth's atmosphere and ionosphere at an angle less than $\sim 60^{\circ}$ of the occultation plane out of the LEO orbital plane. The LEO satellite thus saw $\sim 16$ (of a total 24) GPS occultations per revolution, where most of those are defined to be "setting" occultations based on the absolute azimuth angle between $135-180^{\circ}$ and the rest are "grazing" occultations for the absolute azimuth angle between $45-135^{\circ}$, and, with nearly 14.6 revolutions per day, collected $\sim 230$ daily occultations and more than forty thousand radio occultation profiles of the Earth's atmosphere and ionosphere properties from April 1995 to Feb. 1997. Actually, the baseline length of the limb-viewing path between an occulting LEO position and its calibration position is increased from top to bottom of occultation; the baseline angle to the center of the Earth is increased from zero to approximately $60^{\circ}$ while the ray perigee descends from orbit height to the Earth's surface within each occultation observation. We note that the projected area of calibrated limb-viewing paths for each occultation on to the LEO orbital plane is partially overlapped by other occultation areas (zero to five or more occultations). For the purpose of considering horizontal gradient and/or inhomogeneity effects in the GPS/MET ionospheric electron density retrieval we can combine data from close-up occultations that take place within a targeted occultation area. We first retrieve the ionospheric electron density profile for each occultation measurement using the Abel inversion and assume the derived electron densities to be the values at a set of limb-viewing paths' tangent point positions which can also be accurately determined from known GPS and LEO satellite positions. The retrieved electron densities at nearby occultations can then provide horizontal gradients by interpolating from cubic spline fit at specified altitudes. Figure 2 shows side view of five close-up occultation observations, where, during each occultation span, occulting-to-calibrating point paths appear as a dense set of lines parallel to the LEO orbital plane. In the original Abel inversion process, the ionospheric electron density is assumed local spherical symmetry and to be a function of the radial distance only as $n_{e 0}(r)$ during each occultation measurement. The calibrated path TECs can be calculated by the GPS-to-LEO TEC difference between the occulting points and the corresponding auxiliary points as described in the previous section, and are also equal to the line integral of retrieved $n_{e 0}(r)$ from the Abel inversion process. However, in this study we further define another ionospheric electron density to be expressed by a two-dimensional function $n_{e 1}(r, \varphi)$ of the radial distance $r$ and the angle $\varphi$ of a point along the transmitter-receiver line-of-sight and the corresponding tangent point to the Earth's center during each occultation measurement, and $n_{e 1}(r, \varphi)$ can be determined by the interpolation of a cubic spline fit to a set of close-up ionospheric profiles [i.e., a set of close-up $n_{e 0}(r)$ ], which have first been retrieved by the Abel inversion to different occultation observations. We note again that, taking into account the horizontal gradient and inhomogeneity of the ionosphere, applying the calibrated TECs directly to the Abel inversion (assuming local spherical symmetry) could induce error and should be "compensated" by values of the difference between the tangent point electron density and the real electron densities along the occultation rays. Of course, the real electron densities can 


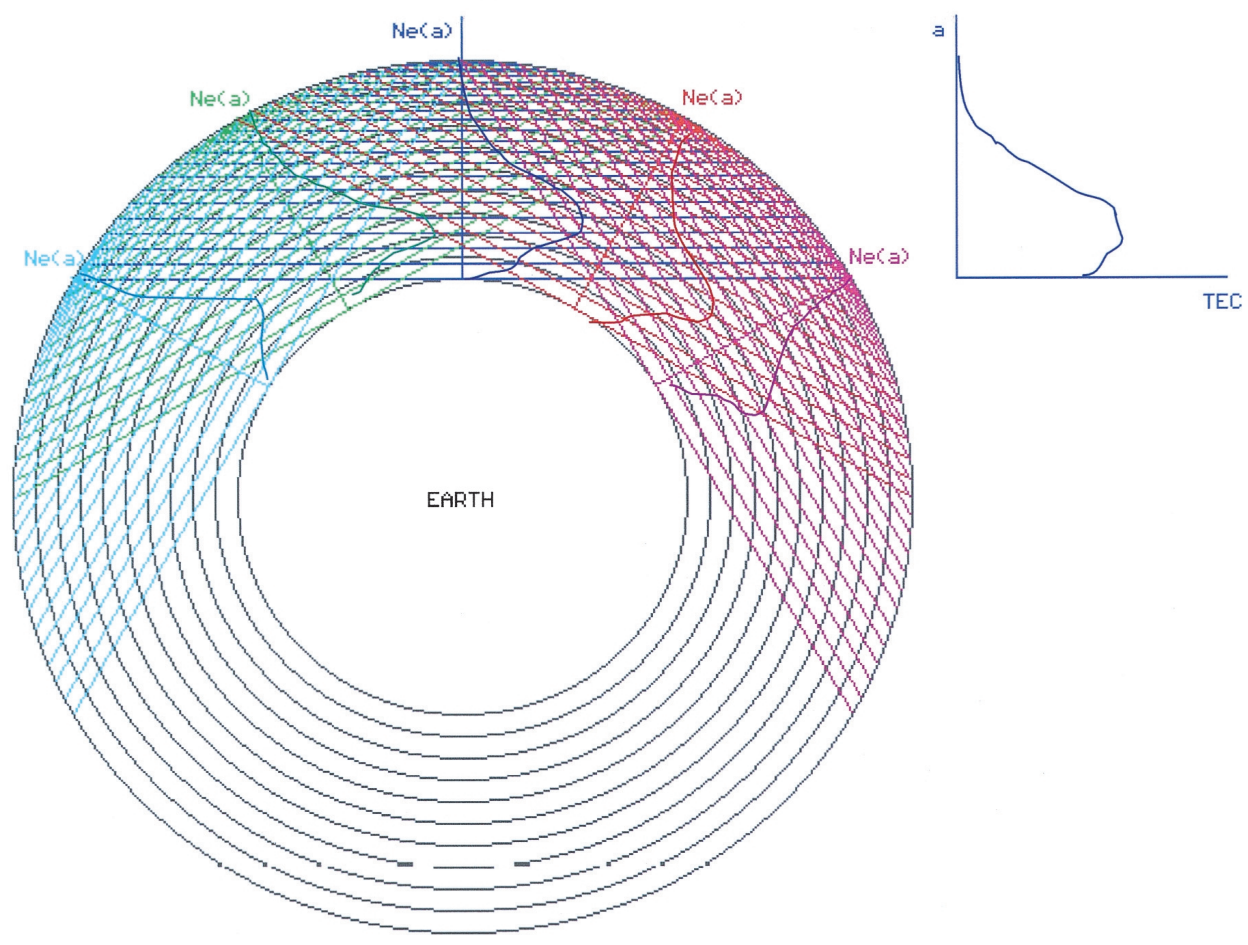

Fig. 2. Sideview of five close-up occultation observations and their retrieved electron density profiles.

not be obtained accurately but are replaced by $n_{e 1}(r, \varphi)$ in this study as described above. The Abel inversion process can now be applied to the "compensated" path TECs, which are defined to be the originally determined path TECs but compensated by the line integral of the electron density differences between $n_{e 0}(r)$ and $n_{e 1}(r, \varphi)$ in the following:

$$
T E C^{\prime \prime}=T E C^{\prime}+\int_{P 1}^{P 2^{\prime}}\left[n_{e 0}(r)-n_{e 1}(r, \varphi)\right] d l,
$$

where $\mathrm{P} 2$ ' is the corresponding calibration position of a LEO satellite position, $\mathrm{P} 1$, with the same radial distance along the transmitter-receiver line-of-sight. We note that $n_{e 0}(r)$ can be updated when the Abel inversion is applied on the compensated path TEC values but not the original calibrated TECs, and further grouped electron density profiles can be interpolated again to derive new $n_{e 1}(r, \varphi)$ and be used to calculate new compensated path TECs. Such procedures could be repeated to approach a reliable result. 
The improved Abel inversion process has been used to remove the effect of local spherical symmetry, i.e., to include the effect of large-scale horizontal gradients, using close-up and pre-determined electron density profiles, which approaches a better result. The large-scale consideration is obtained from the distribution density of occultation observations, which is $\sim 20^{\circ}$ on average to the center of the Earth between neighboring occultation observations. To clarify more details of the improved Abel inversion scheme, the main process steps are listed as the following:

(1) Interpolate measured ionospheric delays (excess phases) at even-spaced radial distances using both GPS L1 and L2 frequencies and determine the GPS-to-LEO TECs.

(2) Determine the occulting LEO positions and the corresponding auxiliary LEO positions with the same impact distances in step (1) and calculate the GPS-to-LEO TEC differences to be the calibrated path TECs as given in Equation (1).

(3) Use the Abel inversion method on the calibrated TECs under the assumption of straightline propagation and local spherical symmetry to retrieve ionospheric electron density profile $\left[n_{e 0}(r)\right]$ and then save the results.

(4) Use close-up nearby electron density profiles within a certain occultation area and interpolate the electron densities $\left[n_{e 1}(r, \varphi)\right]$ along the lines of sight for this targeted occultation.

(5) Calculate the compensated path TECs from the interpolated electron densities along the lines of sight, i.e., the straight-line paths between the occulting points and the calibration points, as given in Equation (2).

(6) Apply the Abel inversion process again on the compensated TECs to retrieve another electron density profile and update $n_{e 0}(r)$.

(7) Steps (4) to (6) can be repeated to approach stable $n_{e 0}(r)$.

An associated flowchart summary is presented in Fig. 3.

\section{4. $f_{0} F_{2}$ COMPARISONS WITH IONOSONDE MEASUREMENTS}

In order to assess the accuracy of the GPS/MET electron density retrievals, we compare the critical frequency estimates of the F2 peak (i.e., $f_{0} F_{2}$ ) from GPS/MET occultation observations with a low-latitude digisonde located at Chung-Li, Taiwan $\left(24.6^{\circ} \mathrm{N}, 121.0^{\circ} \mathrm{E}\right)$. It has been shown that ionosonde measurements can provide accurate estimate of the critical ionospheric plasma frequency from ionogram analyses (Piggott and Rawer 1978; Paul and Mackison 1981). For comparison with the Chung-Li digisonde, we collected 232 matches out of 403 occultations from 217 days (during ten prime times from October 11, 1995, to February 23, 1997, in the GPS/MET mission) where the tangent point location of at the F2 peak of each occultation is within $\pm 12^{\circ}$ of the Chung-Li digisonde site in latitude and longitude. Operation of the Chung-Li digisonde was responsible for loss of most missed matches. However, several of the missed matches were caused by strong sporadic E layer reflection, hence no $f_{0} F_{2} s$ were scaled in the ionogram process. Figure 4 shows the corresponding statistical comparison of derived $f_{0} F_{2}$ from the GPS/MET experiment using the original Abel inversion through the calibrated TECs and the digisonde measurements. The upper-left panel in Fig. 4 illustrates a scatter plot and the corresponding least-squares fitting line for the GPS/MET $f_{0} F_{2}$ values 


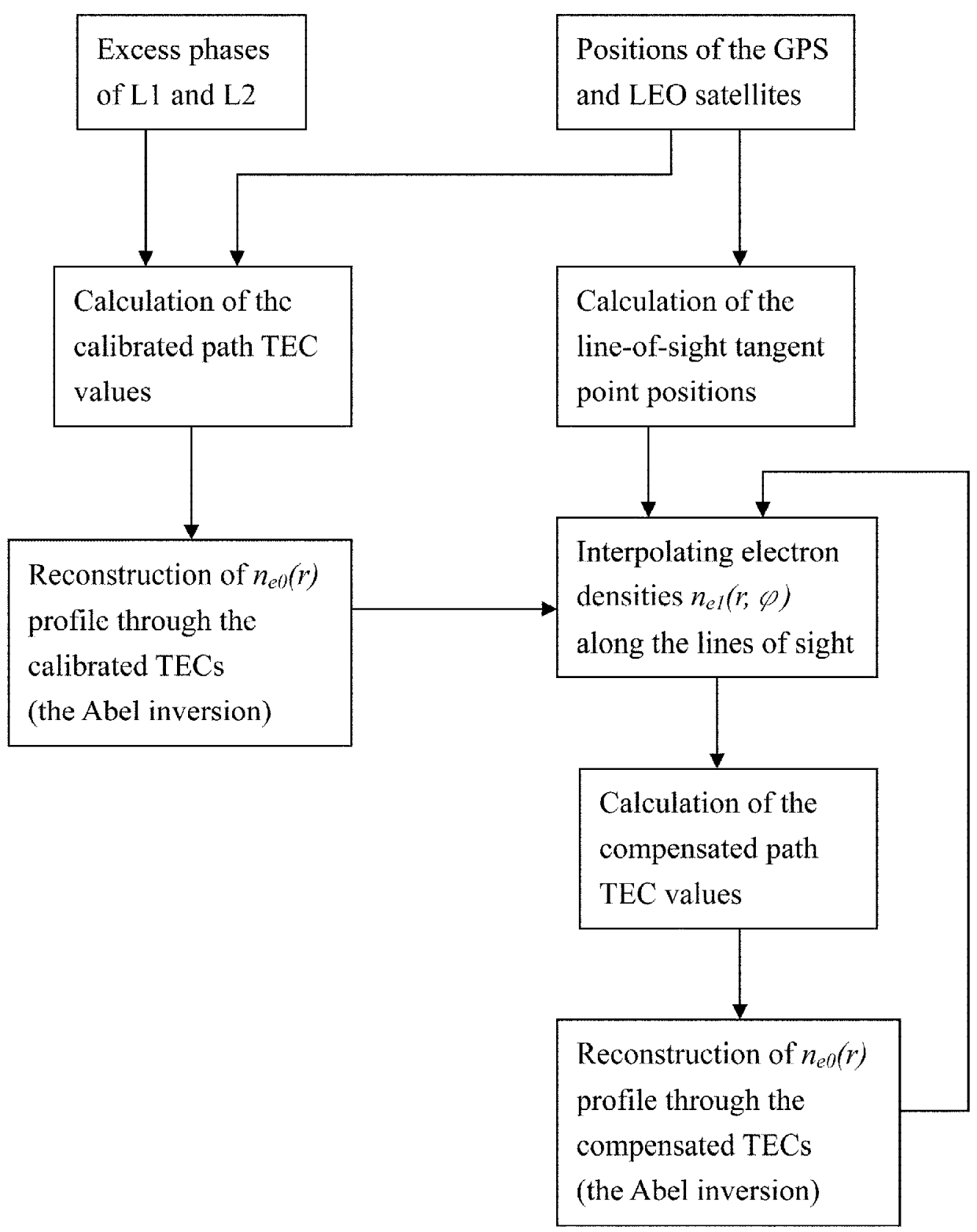

Fig. 3. A flowchart summarizing the improved Abel inversion scheme through the compensated TEC values. 

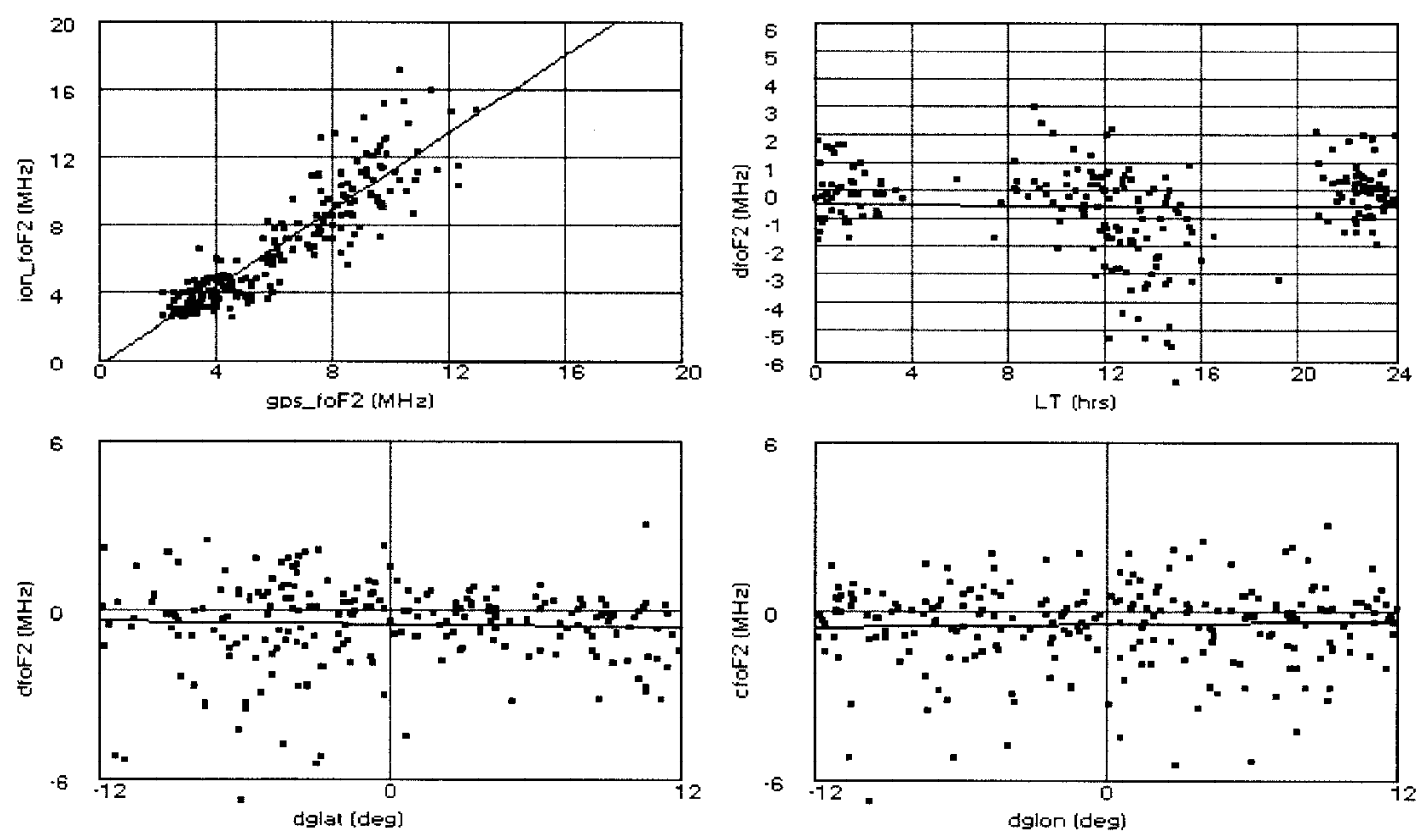

Fig. 4. The upper-left panel illustrates a scatter plot of $f_{0} F_{2}$ observed from GPS/ MET occultation measurements using the original Abel inversion through calibrated TECs and ionosonde measurements above Chung-Li $\left(24.6^{\circ} \mathrm{N}\right.$, $\left.121.0^{\circ} \mathrm{E}\right)$, Taiwan. The upper-right panel, lower-left panel, and lowerright panels illustrate scatter plots of the associated $f_{0} F_{2}$ differences in functions of local time, latitude difference, and longitude difference, respectively.

versus the averaged $f_{0} F_{2}$ values of the associated digisonde measurements in $\pm 0.5 \mathrm{hr}$ of each occultation observing time. The results have a high linear correlation coefficient of 0.90 and a fitting line with a slope of 1.14 and a negative bias of $-0.35 \mathrm{MHz}$. The corresponding $r m s f_{0} F_{2}$ difference is $1.58 \mathrm{MHz}$. We next turn to measures of association between the $f_{0} F_{2}$ difference and other variables of local time and location difference. The upper-right panel, lower-left panel, and lower-right panel illustrate scatter plots and their least-squares fitting lines of the corresponding $f_{0} F_{2}$ differences in functions of local time, latitude difference, and longitude difference, respectively. It is noted that, for the overhead ionosphere above Chung- $\operatorname{Li}\left(24.6^{\circ} \mathrm{N}\right.$, $121.0^{\circ} \mathrm{E}$ ), there is not much association of the $f_{0} F_{2}$ difference to either local time (the upperright panel), the latitude difference (the lower-left panel), or the longitude difference (the lowerright panel) with linear correlation coefficients of $-0.02,-0.04$, and 0.04 , respectively. However, as seen in the scatter plot of the $f_{0} F_{2}$ difference versus local time, the $f_{0} F_{2}$ values retrieved by the GPS/MET occultation observations are under-determined at a ratio of $>75 \%$ ( 57 out of 72 matches) during 12 AM to 8 PM local time. Such results have been proved to be due to even 
symmetry at crests of the equatorial anomaly (Tsai et al. 2002). It is well known that the electron density at $\sim 20^{\circ}$ latitude (close to $24.6^{\circ} \mathrm{N}$ at Chung-Li) corresponds to a maximum in a plane of occultation within $\sim 20^{\circ} \pm 25^{\circ}$ latitude because of the fountain effect in the equatorial region. Therefore, due to the effect of the spherical symmetry assumption utilized for the Abel inversion, such even symmetry with a maximum center would likely cause the Abel inversion to systematically under-estimate the electron density and even $f_{0} F_{2}$ at the tangent points near the peak densities of the equatorial anomaly.

Another statistical comparison between the digisonde scaled $f_{0} F_{2}$ and the GPS/MET occultation experiment using the improved Abel inversion process (one iteration only) through the compensated TECs is shown in Fig. 5. The upper-left panel in Fig. 5 illustrates another scatter plot of the improved occultation $f_{0} F_{2}$ values versus the digisonde $f_{0} F_{2}$ values and shows a similar linear correlation coefficient value of 0.91 compared to Fig. 4 , but shows better results on the least-squares fitting line (a slope of 1.02) and the corresponding bias $(-0.02 \mathrm{MHz})$. The rms $f_{0} F_{2}$ difference is also improved from $1.58 \mathrm{MHz}$ to $1.41 \mathrm{MHz}$. Furthermore, from the upper-right panel in Fig. 5 of the scatter plot of the $f_{0} F_{2}$ difference versus local time (during $12 \mathrm{AM}$ to $8 \mathrm{PM}$ ), the ratio of under-determined $f_{0} F_{2}$ values by the GPS/MET occultation observations is improved to $62 \%$ (45 out of 72 matches). The upperright and lower-right panels still illustrate no correlative relationship between the $f_{0} F_{2}$ differences to local time and longitude differences, respectively. However, in the lower-left panel of
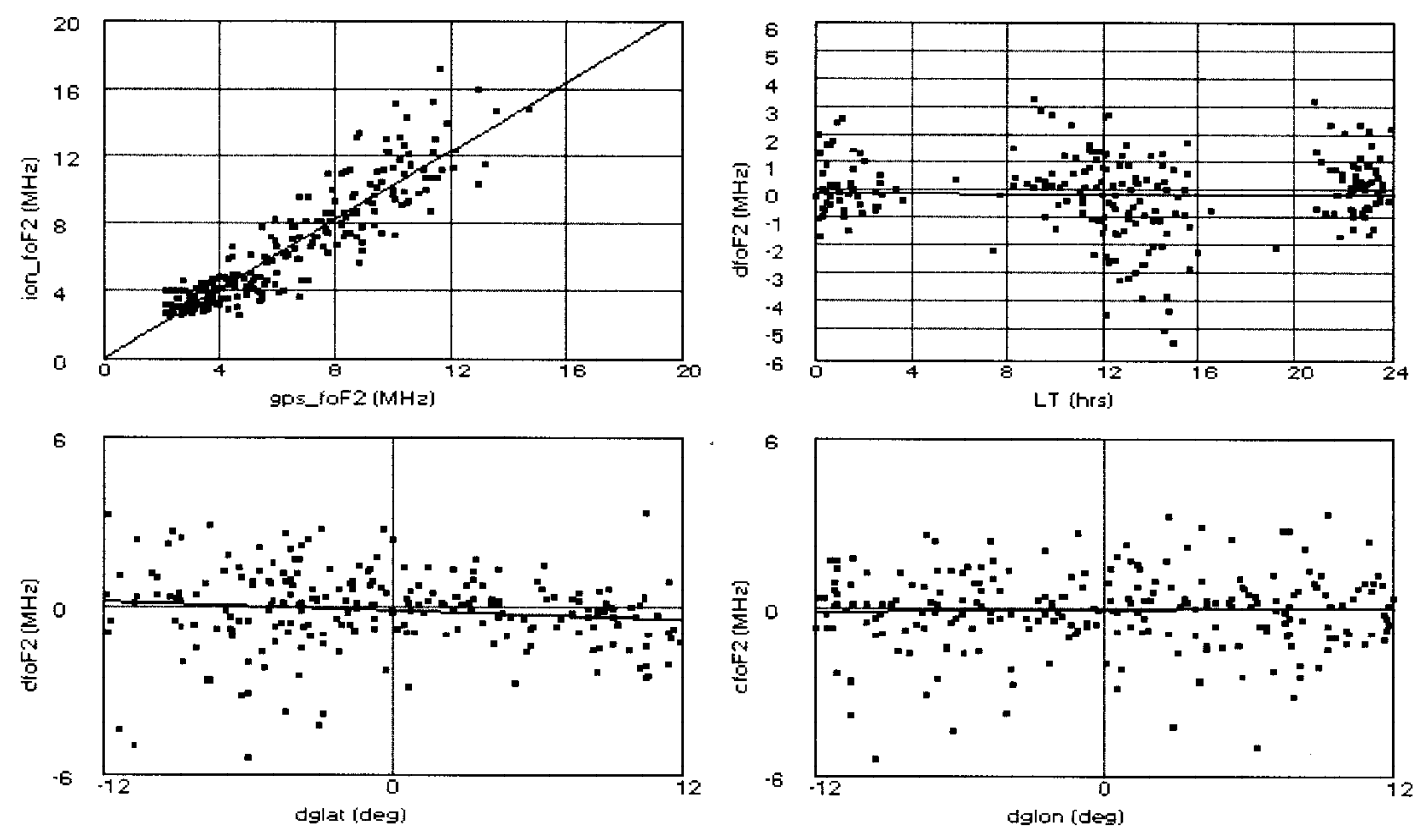

Fig. 5. Same as Fig. 4 for the GPS/MET $f_{0} F_{2}$ retrieved by the improved Abel inversion process through compensated TECs. 
Fig. 5, there presents a more negative association with a linear correlation coefficient of -0.12 between the $f_{0} F_{2}$ difference and the latitude difference from the GPS/MET measurement to the ionosonde measurement. The fitting line's slope at $-0.03 \mathrm{MHz}$ per degree in latitude (with a bias of $-0.12 \mathrm{MHz}$ ) denotes higher latitude having lower critical frequency. Figure 6 also shows plots of plasma frequency profiles that are retrieved from calibrated and compensated TECs. The purpose of this comparison is to show that the electron density profiles through compensated TECs sometimes present more noticeable information, e.g., an E layer in this example.

We note that the Microlab-1 spacecraft was equipped with only one antenna in view of setting GPS satellites at an angle less than $\sim 60^{\circ}$ out of the LEO orbital plane. The LEO orbit and GPS-to-LEO occultation links are almost not coplanar and introduce an absolute azimuth angle of $<60^{\circ}$ from the LEO satellite. In our analyses, such azimuth angles have no correlative relationship to the derived $f_{0} F_{2}$ differences of ionosonde data versus either Abel inversion approach using calibrated TECs or compensated TECs. This result matches the correlative comparisons of the $f_{0} F_{2}$ difference with the longitude difference as shown in the lower-left panels of Figs. 4 and 5. On the other hand, in the improved Abel inversion process, we define a grouped occultation number to be the number of occultation observations where the LEO-

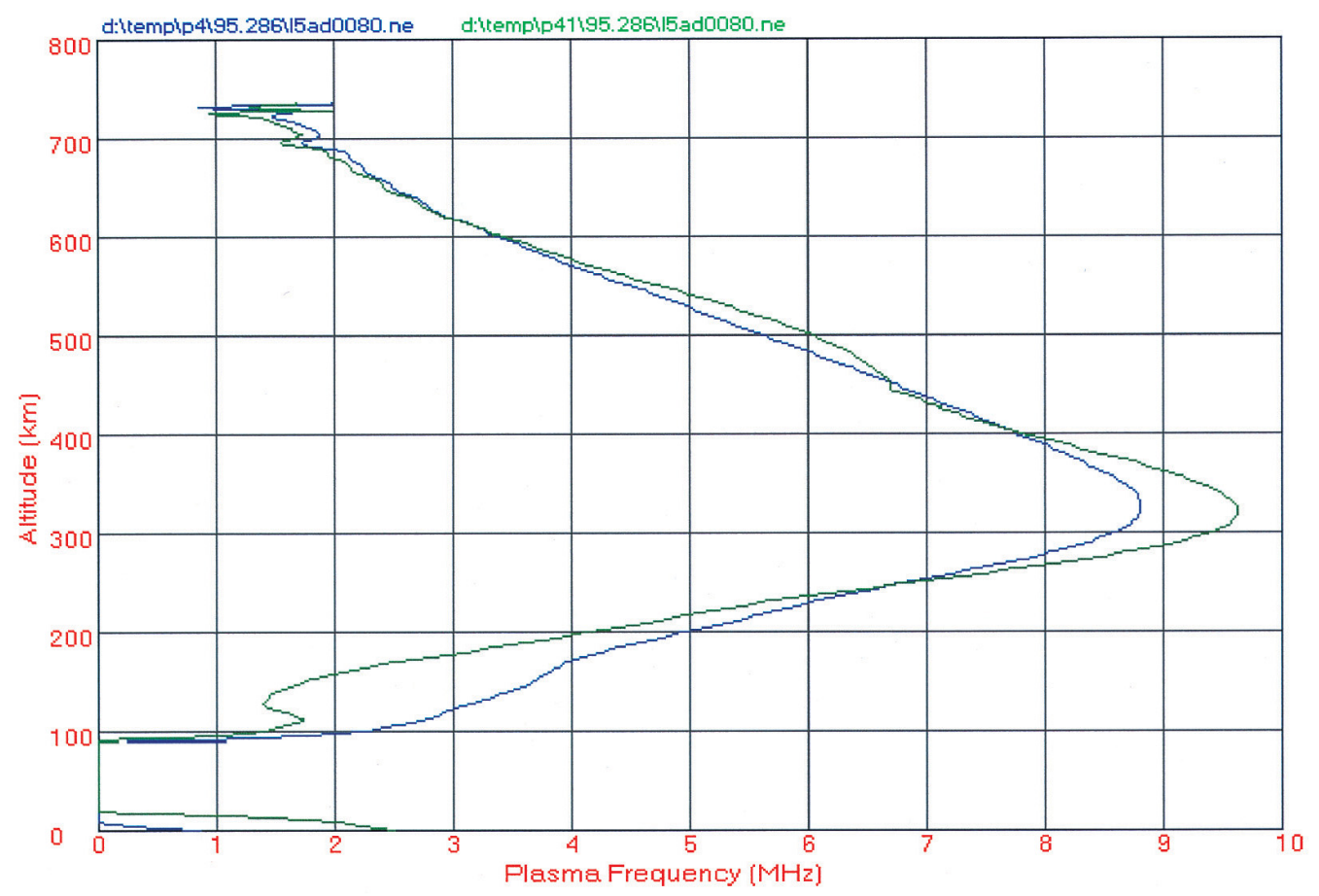

Fig. 6. Example of Abel inversions using calibrated (blue line) and compensated (green line) TECs on October 13, 1995. 
orbital latitudes of their peak densities are located within the bottom baseline region of the targeted occultation. We note that, in the GPS/MET mission, the grouped occultation number is usually $\leq 5$ because the bottom baseline angle between the occulting LEO position and the corresponding calibration position to the center of the Earth is about $60^{\circ}$ and there are $\sim 17$ occultations during one revolution of the LEO satellite, i.e., $\sim 20^{\circ}$ on average between occultations. The grouped occultation number could be one (the targeted occultation itself) only when the targeted occultation is a grazing occultation. Table 1 lists the occultation distribution under different grouped occultation numbers over the searched 232 matches and their corresponding $r m s f_{0} F_{2}$ difference changes (compared with the original $r m s f_{0} F_{2}$ difference using calibrated TECs) after the $1^{\text {st }}$ iteration and $2^{\text {nd }}$ iteration processes of the improved Abel inversion scheme. It is clearly shown that more grouped occultations within the targeted occultation area improve GPS/MET $f_{0} F_{2}$ retrieval. The improved Abel inversion process using compensated TEC values cannot approach better $f_{0} F_{2}$ estimate when the grouped occultation number is $\leq 3$ but there is significant improvement with numbers larger than 3 . This result is as expected because more occultations within the targeted area can be used to interpolate more accurate electron densities and then compensate path TECs. Furthermore, as shown in Table 1, both $f_{0} F_{2}$ retrievals after the $1^{\text {st }}$ and $2^{\text {nd }}$ iteration of the improved Abel inversion process show improvement (negative $r m s \quad f_{0} F_{2}$ difference changes) on the original Abel inversion through calibrated TECs, but the previous retrievals present better results. The reason could be explained by the lack of enough close-up occultations in the GPS/MET ionospheric data.

\section{VALIDATION THROUGH THE REFERENCE GPS/MET EXPERIMENT USING THE IRI}

Except for specified overhead ionosphere above the ground-based validation stations, e.g., ionosondes, it is difficult to obtain the true ionosphere used to verify retrieved electron

Table 1. The distribution and their corresponding $r m s \Delta f_{0} F_{2}$ changes under different grouped occultation numbers for the searched 232 matches to the Chung-Li digisonde data.

\begin{tabular}{|l|l|l|l|l|}
\hline $\begin{array}{l}\text { Grouped Occultation } \\
\text { Number }\end{array}$ & $\begin{array}{l}\text { Number } \\
\text { Matches }\end{array}$ & $\begin{array}{l}r m s f_{o} F_{2} \text { Changes in } \\
M H z\left(1^{\text {st }} \text { iteration }\right)\end{array}$ & $\begin{array}{l}r m s f_{o} F_{2} \text { Changes in } \\
\mathrm{MHz}\left(2^{\text {nd }} \text { iteration }\right)\end{array}$ \\
\hline 1 & 10 & 0.26 & 0.24 \\
\hline 2 & 44 & -0.02 & -0.01 \\
\hline 3 & 82 & -0.04 & 0.07 \\
\hline 4 & 72 & -0.30 & -0.28 \\
\hline 5 & 23 & -0.47 & -0.46 \\
\hline 6 & 1 & -1.68 & -1.68 \\
\hline Summary & 232 & -0.17 & -0.12 \\
\hline
\end{tabular}


densities within occultation observations. Furthermore, validation using ground-based observation raises the problems of spatial and/or temporal mismatch to actual occultation observations as discussed in the previous section. In the following, we simulate the path TECs by integrating the IRI-modeled electron densities along the straight paths between the LEO satellite positions and their corresponding auxiliary positions obtained during the actual GPS/MET experiment, where the GPS/MET LEO orbit estimation has been performed with GPS triple difference measurements and can approach a precise position of $\sim 15 \mathrm{~cm} 3 \mathrm{D}$ root mean square (Schreiner et al. 1998). The simulated path TECs have been computed by numerical integration Gaussian Quadrature of order 7 over an interval of $50 \mathrm{~km}$ along the straight paths. New one-dimensional electron density profiles can then be retrieved again by both of the original Abel inversion method through calibrated IRI-modeled TECs and the improved 7-step Abel inversion scheme through further compensated IRI-modeled TECs. Such simulation is called the "reference" GPS/MET experiment in this paper. The retrieved electron density profiles can then be compared and verified by the "true" IRI-modeled ionosphere.

The IRI model is not a theoretical model but an empirical standard ionosphere model based on many data sources including the worldwide network of ionosondes, the powerful incoherent scatter radars (Jicamarca, Arecibo, Millstone Hill, Malvern, St. Santin), the ISIS and Alouette topside sounders, and in situ instruments on several satellites and rockets (Rawer et al. 1978, 1978, 1981; Bilitza 1990, 2001). The IRI model was developed more than 30 years ago and has achieved a quite realistic description of highly variable ionosphere; several steadily improved editions of the model have been released from 1969 to 2001. The aim of the IRI model is to establish a summary compendium of height profiles through the ionosphere for the four main plasma parameters: plasma density, electron temperature, ion plasma temperature, and ion composition. In this paper, we focus on plasma density only for GPS-to-LEO path TEC simulation. We use the IRI 2001 model of monthly averages in the non-auroral ionosphere for magnetically quiet conditions. Other extensions of disturbed ionosphere can be planned under the conditions of the ion drift model, description of the auroral and polar ionosphere, and magnetic storm effects.

In this section we statistically compare and verify the original Abel inversion and the improved 7-step Abel inversion scheme using the "true" IRI-modeled data within the reference GPS/MET experiment. We have processed all GPS/MET ionospheric observations (more than forty thousand records) within 13 prime times ( 250 days) from April 1995 to Feb. 1997. Approximately $75 \%$ of occultation observations within the GPS/MET experiment can be retrieved into one-dimensional electron density profiles at the limb-viewing paths' tangent points. The major reasons for failures in electron density retrieval are insufficient data and large time gaps on receiving the targeted GPS satellite signals at the occulting and/or calibrating sides. Such failures also happen within the reference GPS/MET experiment because no GPS and LEO satellite position data was recorded and thus lack TEC simulation. Figure 7 shows a scatter plot of derived $f_{0} F_{2}$ (referred to the left y-axis) using the original Abel inversion through the calibrated TECs versus the "true" IRI-modeled $f_{0} F_{2}$ (referred to the x-axis) and a fractional mean $f_{0} F_{2}$ difference curve (referred to the right y-axis) as a function of the true IRImodeled $f_{0} F_{2}$ also (shown in the light-blue line). As discussed in the previous sections, the Abel inversion uses the assumption of local spherical symmetry of ionospheric electron den- 
sity and has no horizontal gradients. This assumption generally provides very good results for electron density inversions as shown as Fig. 7, where most derived $f_{0} F_{2}$ using the original Abel inversion are within a 10\% fractional mean difference comparable to the true IRI-modeled $f_{0} F_{2}$. If there are small-scale structures or constant (nonzero) horizontal gradients in the real electron density distribution, the horizontal inhomogeneity can be smeared out during the inversion since errors introduced by the odd terms will cancel upon integrating along the ray path. However, as seen from Fig. 7, retrieved values of $f_{0} F_{2}$ are biased high, i.e., overestimated, with respect to the true IRI-modeled $f_{0} F_{2}$ with a maximum $20 \%$ mean difference at a minimal $\sim 1 \mathrm{MHz} f_{0} F_{2}$, but such positive biases are decreased and less than $5 \%$ for $f_{0} F_{2}$ values between $2.5 \mathrm{MHz}$ and $8 \mathrm{MHz}$. On the other high $f_{0} F_{2}$ side, retrieved values are biased low (underestimated) with respect to the IRI-modeled data when $f_{0} F_{2} s$ are larger than $8 \mathrm{MHz}$. The underestimated condition can be approached to a maximum $\sim 15 \%$ mean difference for $f_{0} F_{2}$

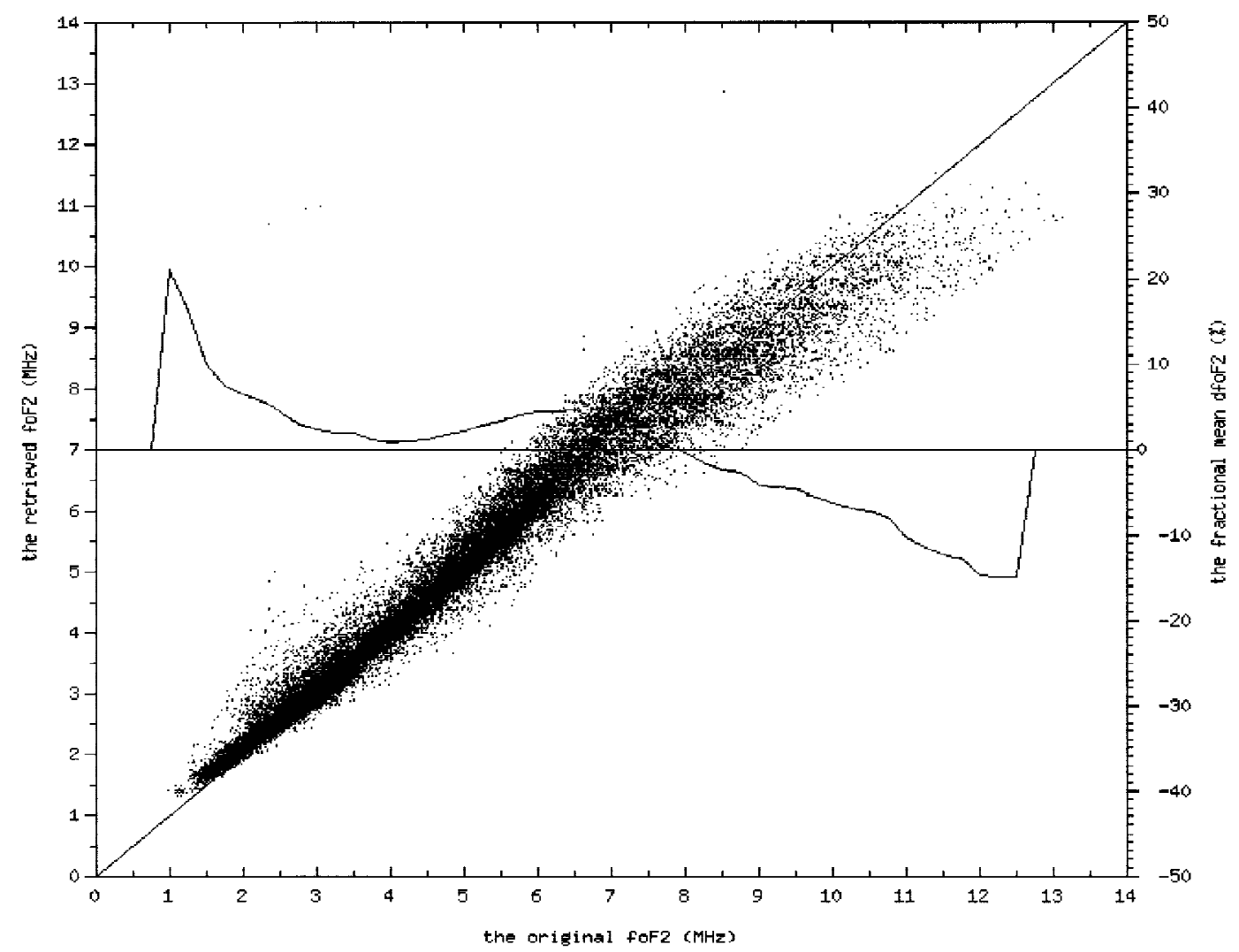

Fig. 7. Plot of the retrieved occultation values of $f_{0} F_{2}$ (using the original Abel inversion method through calibrated TECs) versus the "true" IRI-modeled values within the reference GPS/MET experiment. The solid line shows the fractional mean difference curve. 
values larger than $12 \mathrm{MHz}$. Either overestimated or underestimated conditions can be typically explained by that ionospheric electron densities are distributed under even symmetry as a valley or crest within the targeted occultation area with the tangent points at a locally minimum or maximum center, respectively. The valley (crest) would be spread out after the Abel inversion and the retrieved electron densities at the tangent points are thus larger (smaller) than the real values. Reasonably, there is a greater possibility low $f_{0} F_{2}$ (high $f_{0} F_{2}$ ) existing under even electron density symmetry with a minimum (maximum) center and to being overestimated (underestimated), as the results shown in Fig. 7.

Another statistical comparison between the improved Abel inversion process (one iteration only) and the IRI-modeled data is shown in Fig. 8. The overestimated conditions of retrieved $f_{0} F_{2}$ between $1 \mathrm{MHz}$ to $2.5 \mathrm{MHz}$ are significantly improved from a maximum fractional mean difference of $\sim 20 \%$ to less than $10 \%$. On the other high $f_{0} F_{2}$ side, improvement of

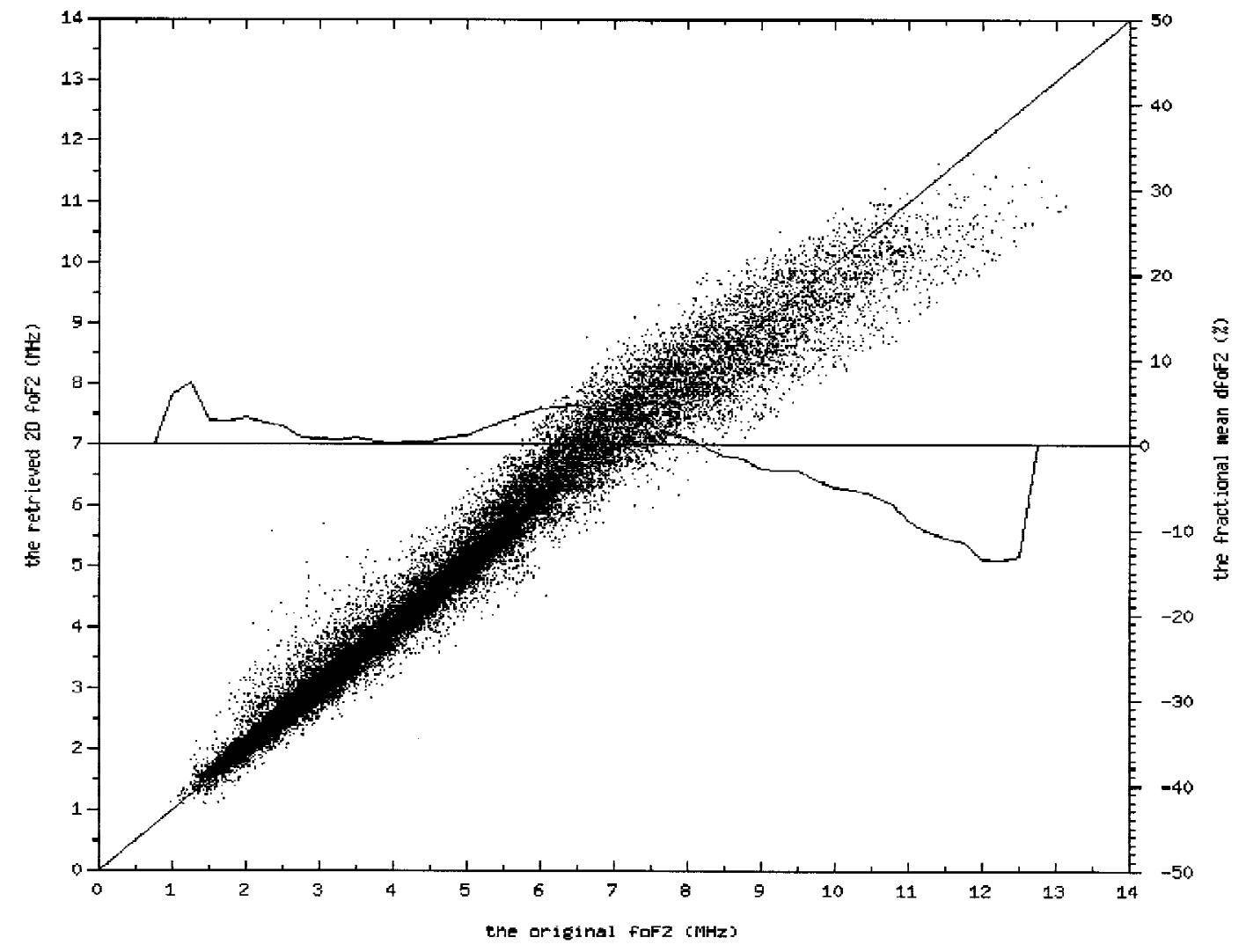

Fig. 8. Same as Fig. 7 for the occultation $f_{0} F_{2}$ retrieved by the improved Abel inversion process through compensated TECs within the reference GPS/ MET experiment. The light-blue line shows another fractional mean difference curve. 
underestimated conditions is not significant but it has a fractional mean difference of $\sim 10 \%$. Table 2 depicts the occultation distribution under different grouped occultation numbers within the reference GPS/MET experiment (totally 38255 observations) and their corresponding fractional $\mathrm{rms} f_{0} F_{2}$ difference compared to the "true" IRI-modeled $f_{0} F_{2}$. The results also prove that more grouped occultations within the targeted occultation area better GPS/MET $f_{0} F_{2}$ retrieval through the improved Abel inversion process using compensated TEC values as discussed in the previous section.

Table 2. The distribution and their corresponding fractional $r m s \Delta f_{0} F_{2}$ under different grouped occultation numbers within the reference GPS/MET experiment.

\begin{tabular}{|l|l|l|l|}
\hline $\begin{array}{l}\text { Grouped } \\
\text { Occultation Number }\end{array}$ & $\begin{array}{l}\text { Number of } \\
\text { Occultations }\end{array}$ & $\begin{array}{l}\text { the original } r m s \\
\Delta f_{0} F_{2} \text { in } \%\end{array}$ & $\begin{array}{l}\text { the improved } r m s \\
\Delta f_{0} F_{2} \text { in } \%\end{array}$ \\
\hline 1 & 1490 & 6.09 & 6.07 \\
\hline 2 & 6043 & 6.43 & 6.34 \\
\hline 3 & 13011 & 6.23 & 5.95 \\
\hline 4 & 12225 & 6.08 & 5.73 \\
\hline 5 & 4706 & 6.07 & 5.67 \\
\hline 6 & 780 & 6.10 & 5.36 \\
\hline Summary & 38255 & 6.19 & 5.90 \\
\hline
\end{tabular}

\section{SUMMARY AND DISCUSSION}

In order to correct the error due to the spherical symmetry assumption used in the Abel inversion, the "constrained-gradient" inversion scheme developed by Straus (1999) assumed the horizontal inhomogeneity of electron densities along the line of sight to be a function of $\varphi$ only. They assumed a two-dimensional ionospheric density to be a product of $n_{e}(r, 0)$, the electron density profile at the tangent point, and $f(\varphi)$, a function which provides the ratio of electron densities along the line of sight to the densities at the tangent point. Actually, such horizontal gradients should be not just function of $\varphi$ but of radial distance as well. Furthermore, the constrained-gradient factor described in Straus (1999) was proposed to be obtained from other ancillary data, e.g., in-situ plasma density, nadir-viewing extreme ultraviolet airglow, and total electron content maps derived from ground-based GPS receivers, but not obtained from occultation measurement itself. In this study real and simulated GPS/MET occultation data have been inverted to ionospheric profiles using the Abel inversion method from two approaches of what we call calibrated TECs and compensated TECs. $f_{0} F_{2} s$ retrieved from both methods have been examined through the same nearby ionosonde data and the reference GPS/MET experiment on the IRI-modeled ionosphere, and the examination results show improved agreement when the grouped occultation number is larger than 3. It is further represented that more grouped occultations within the targeted occultation area can approach better 
occultation $f_{0} F_{2}$ retrieval. We note that the Microlab-1 spacecraft was equipped with only one antenna and thus saw $\sim 16$ (of a total 24) occultations per revolution. In future work, placing one more occultation antenna on GPS receivers in view of rising GPS in low-Earth orbit, such as the Constellation Observing System for Meteorology Ionosphere and Climate (COSMIC) mission, could provide twice the number of occultations to determine more accurate interpolated electron densities in the improved Abel inversion scheme. Thus more accurate ionospheric profiling can be achieved through more accurate compensated TEC values.

Acknowledgements We are grateful to Dr. W. S. Schreiner and D. C. Hunt (UCAR) who provided the $2^{\text {nd }}$ level ionospheric data of the GPS/MET experiment. This work has been supported by Projects NSC92-NSPO(B)-RS3-FA07-01D and NSC91-2111-M008-033.

\section{REFERENCES}

Bilitza, D. (Ed.), 1990: International Reference Ionosphere 1990, NSSDC 90-22, Greenbelt, Maryland.

Bilitza, D., 2001: International Reference Ionosphere 2000. Radio Sci., 36, 261-275.

Dymond, K. F., and R. J. Thomas, 2001: A technique for using measured ionospheric density gradients and GPS occultations for inferring the nighttime ionospheric electron density. Radio Sci., 36, 1141-1148.

Fjeldbo, G., and V. R. Eshleman, 1969: Atmosphere of Venus as studied with the Mariner V dual radio frequency occultation experiment . Radio Sci., 4, 879-897.

Hajj, G. A., and L. J. Romans, 1998: Ionospheric electron density profiles obtained with the Global Positioning System: Results from the GPS/MET experiment. Radio Sci., 33, 175-190.

Hajj, G. A., L. C. Lee, X. Pi, L. J. Romans, W. S. Schreiner, P. R. Straus, and C. Wang, 2000: COSMIC GPS ionospheric sensing and space weather. TAO, 11, 235-272.

Hocke, K., and K. Igarashi, 2002a: Structure of the Earth's lower ionosphere observed by GPS/MET radio occultation. J. Geophys. Res., 107, 10.1029/2001JA900158.

Hocke, K., and K. Igarashi, 2002b: Electron density in the F region derived from GPS/MET radio occultation data and comparison with IRI. Earth Planets Space, 54, 947-954.

Kilore, A. J., D. L. Cain, G. S. Levy, V. R. Eshlemann, G. Fjeldbo, and F. D. Drake, 1965: Occultation experiment: Results of the first direct measurement of Mars' atmosphere and ionosphere. Science, 149, 1243-1248.

Kursinski, E. R., G. A. Hajj, J. T. Schofield, R. P. Linfield, and K. R. Hardy, 1997: Observing Earth's atmosphere with radio occultation measurements using the Global Positioning System. J. Geophys. Res., 102, 23429-23465.

Paul, A. K., and D. L. Mackison, 1981: Scaling of the F-layer critical frequency from digital ionograms applied to observations during the solar eclipse on 26 February 1979.J. Atmos. Terr. Phys., 43, 221-223. 
Piggott, W. R., and K. Rawer, 1978: U.R.S.I. Handbook of Ionogram Interpretation and Reduction, $2^{\text {nd }}$ Edition, World Data Center A for Solar-Terr. Physics, Report UAG$23 \mathrm{~A}$.

Rawer, K., D. Bilitza, and S. Ramakrishnan, 1978: Goals and status of the International Reference Ionosphere. Rev. Geophys., 16, 177-181.

Rawer, K., S. Ramakrishnan, and D. Bilitza, 1978: International Reference Ionosphere 1978, International Union of Radio Science, URSI Special report, Bruxells, Belgium, 75 pp.

Rawer, K., J. V. Lincoln, and R. O. Conkright, 1981: International Reference Ionosphere-IRI 79, World Data Center A for Solar-Terrestrial Physics, Report UAG-82, Boulder, Colorado, 245p.

Reilly, M. H., 1993: A transionospheric radio propagation model, Proc. Ionospheric Effects Symp., Alexandria, VA, May 4-6.

Rius, A., G. Ruffini, and L. Cucurull, 1997: Improving the vertical resolution of ionospheric tomography with GPS occultations. Geophys. Res. Lett., 24, 2291-2294.

Rius, A., G. Ruffini, and A. Romeo, 1998: Analysis of ionospheric electron density distribution from GPS/MET occultations. IEEE Trans. Geosci. Remote Sens., 36, 383-394.

Rocken, C., R. Anthes, M. Exner, D. Hunt, S. Sokolovskiy, R. Ware, M. Gorbunov, W. Schreiner, D. Feng, B. Herman, Y. Kuo, and X. Zou, 1997: Analysis and validation of GPS/MET data in the neutral atmosphere. J. Geophys. Res., 102, 29849-29866.

Schreiner, W. S., D. C. Hunt, C. Rocken, and S. V. Sokolovskiy, 1998: Precise GPS data processing for the GPS/MET radio occultation mission at UCAR, in Proc. Inst. Navigation-Navigation 2000, Alexandria, Va., 103-112.

Schreiner, W. S., S. V. Sokolovskiy, C. Rocken, and D. C. Hunt, 1999: Analysis and validation of GPS/MET radio occultation data in the ionosphere. Radio Sci., 34, 949-966.

Sokolovskii, S. V., 1990: Solution of the inverse refraction problem by sensing of the atmosphere from space. Sov. J. Remote Sens., 6, 333-338.

Straus, P. R., 1999: Correcting GPS occultation measurements for ionospheric horizontal gradients, Proc. Ionospheric Effects Symp., Alexandria, VA, June.

Tricomi, F. G., 1985: Integral Equations. Dover, Mineola, N. Y., 238 p.

Tsai, L. C., W. H. Tsai, W. S. Schreiner, F. T. Berkey, and J. Y. Liu, 2001: Comparisons of GPS/MET retrieved ionospheric electron density and ground-based ionosonde data. Earth Planets Space, 53, 193-205.

Tsai, L. C., C. H. Liu, W. H. Tsai, and C. T. Liu, 2002: Tomographic imaging of the ionosphere using the GPS/MET and NNSS data. J. Atmos. Solar-Terr. Phys., 64, 20032011.

Ware, R., and Coauthors, 1996: GPS sounding of the atmosphere from low Earth orbit: preliminary results. Bull. Am. Meteor. Soc., 77, 19-40.

Zou, X. Y., H. Kuo, and Y. R. Guo, 1995: Assimilation of atmospheric radio refractivity using a nonhydrostatic mesoscale model. Mon. Wea. Rev., 123, 2229-2249. 